\title{
Citizen Science - Die Rolle des Bürgers in der Wissenschaft
}

\author{
Frank Hartmann* und Dana Mietzner
}

\section{Zusammenfassung}

Die Wissenschaft öffnet sich in zunehmendem Maße anderen gesellschaftlichen Teilbereichen und unter dem Schlagwort Citizen Science (Bürgerwissenschaft) verstärkt auch Bürgerinnen und Bürgern. Ein solcher Öffnungsprozess ist, wie die gegenwärtige wissenschaftliche Diskussion zeigt, weder theoretisch noch praktisch unproblematisch. In diesem Beitrag werden deshalb zunächst verschiedene theoretisch-konzeptionelle Ansätze skizziert, die mit dem Thema Bürgerwissenschaft im Zusammenhang stehen, um die unterschiedlichen Facetten der Diskussion zu verdeutlichen. Des Weiteren beabsichtigen die Autorin und der Autor vor allem Wissenschaftlerinnen und Wissenschaftler unterschiedlicher Disziplinen und Forschungsrichtungen für Chancen und Grenzen des Konzepts Bürgerwissenschaft und seiner praktischen Umsetzung in verschiedenen Formen zu sensibilisieren. Schließlich werden vor dem Hintergrund der theoretisch-konzeptionellen Diskussionen Anforderungen an den Aufbau eines bürgerwissenschaftlichen Panels an Hochschulen als mögliches Instrument von Citizen Science spezifiziert.

\begin{abstract}
Science increasingly opens up to other societal subsystems - including citizens - and labeled as Citizen Science (Bürgerwissenschaft). Current scientific discussions show that such an opening process is neither theoretically nor practically without problems. This article therefore presents different theoretical-conceptual approaches, which are related to the topic of citizen science, in order to illustrate the different facets of the discussion. Furthermore, the authors intend to sensitize scientists and scholars of various disciplines and research areas for the opportunities and limitations of the concept of citizen science and its practical implementation in various forms. Finally, against the background of the theoretical-conceptual discussions, requirements for the establishment of a citizen science panel at Institutions of Higher Education are specified as a possible tool of Citizen Science.
\end{abstract}

\section{Einleitung}

Das Innovationsmanagement in der unternehmerischen Praxis hat sich in den vergangenen Jahren von „Open Innovation“ (Öffnung von Innovationsprozessen gegenüber Partnern und externe Verwertung von F\&EErgebnissen und Innovationen) über "Open Local Innovation“ (Öffnung von Unternehmen für die Zusammenarbeit mit kommunalen Verwaltungen) bis hin zu „Open Collaborative Innovation" (Einbezug von Bürgern in Innovationsprozesse) und „Free Innovation“ (Bürger als Innovatoren) entwickelt (Chesbrough 2003: 180; Reichwald \& Piller 2009; Baldwin \& von Hippel 2011; von Hippel 2017).
Im Wissenschaftsbereich zeichnet sich eine ähnliche Entwicklung ab. Während sich die Wissenschaft gegenüber der Wirtschaft längst geöffnet hat, erodieren die Grenzen auch zu anderen gesellschaftlichen Teilbereichen, und die Wissenschaft beginnt nun, sich unter dem Schlagwort „Bürgerwissenschaft" bzw. "Citizen Science“ verstärkt Laien und zivilgesellschaftlich organisierten Bürgerinnen und Bürgern zu öffnen (Wissenschaft im Dialog o. J.). Für Hochschulen mit ihren spezifischen Funktionen und Ressourcen im Wissenschaftssystem ergeben sich hiermit neue Herausforderungen und Chancen, die weiter auszuloten sind.
Dieser Beitrag erhebt nicht den Anspruch einer umfassenden Literaturanalyse, er hat vielmehr den Charakter einer erklärenden und argumentativen Abhandlung mit dem Ziel, eine eigene Perspektive auf den Untersuchungsgegenstand Bürgerwissenschaft (Citizen Science) zu entwickeln. Es werden verschiedene theoretischkonzeptionelle Ansätze skizziert, die mit dem Thema Bürgerwissenschaft im Zusammenhang stehen. Damit solIen (1) unterschiedliche Facetten der Diskussion verdeutlicht, (2) eine Sensibilisierung für Chancen und Grenzen des Konzepts Bürgerwissenschaft erreicht und (3) die Grundlage für die Konzipierung eines Instrumentes geschaffen werden, mit dessen Un- 
terstützung Bürgerinnen und Bürger schrittweise in die Forschung einer Hochschule eingebunden werden können. Dazu werden vor allem theoretische Konzepte zum Thema Open Science und Citizen Science systematisiert, aber auch Ansätze aus dem Bereich der Bürgerbeteiligung als Form der Partizipation an politischen Entscheidungen, hier insbesondere zu Bürgerpanels, betrachtet. Vor diesem Hintergrund werden die Anforderungen an den Aufbau eines bürgerwissenschaftlichen Panels an einer Hochschule spezifiziert, was als Grundlage für den Aufbau und die Moderation des Panels dient.

\section{Konzeptioneller Hintergrund}

In diesem Abschnitt wird die Öffnung des Wissenschaftssystems überwiegend aus der internen Perspektive der Wissenschaft thematisiert. Dabei werden wissenschaftstheoretische und auch wissenschaftspolitische Ansätze (Open Science) aufgegriffen. Weiterhin werden Ansätze vorgestellt, die steigende Partizipationsansprüche von Bürgerinnen und Bürgern zum Ausgangspunkt nehmen, um eine weitere Öffnung des Wissenschaftssystems zu begründen (Citizen Science) (Finke 2014: 208).

Dabei ist zu berücksichtigen, dass zwar einzelne Stränge im wissenschaftlichen Diskurs erkennbar sind, am deutlichsten jener, der sich von den Konzepten „Post Normal Science“ (Funtowicz \& Revetz 1993) und "Mode 2" (Gibbons 1994) bis hin zum Ansatz der "Transformativen Wissenschaft“ (Schneidewind \& Singer-Brodowski 2014) im Jahr 2014 durchzieht. Insgesamt betrachtet ist die Diskussion jedoch sehr vielschichtig, hochaktuell und offen, wie jüngst die Zeitschrift für Technikfolgenabschätzung in Theorie und Praxis mit ihrem Spezialheft zum Thema „Open Science zwischen Hype und Disruption" gezeigt hat (Grunwald 2017).

Schließlich wird in verkürzter Form auf politische Beteiligungsformate verwiesen, insbesondere auf das Format des Bürgerpanels, um auch aus dieser Perspektive Anregungen für den Aufbau eines bürgerwissenschaftlichen Panels an Hochschulen zu gewinnen.

\subsection{Open Science}

Aus wissenschaftstheoretischer und wissenschaftspolitischer Sicht sind es vor allem zwei Arbeiten, die eine breite und anhaltende Diskussion um die moderne Wissenschaft angestoßen haben und mit dem Thema Citizen Science in engem Zusammenhang stehen. Funtowicz und Ravez (1993) lenkten mit ihrem Artikel "Science for the Post-Normal Age“ den Blick auf einen systemischen, synthetischen und humanistischen Ansatz des Verständnisses von Wissenschaft, im Unterschied zu einer von ihnen als reduktionistisch und analytisch bezeichneten Weltsicht, in der immer tiefer spezialisierte Wissenschaftler immer weiter analytisch segmentierte Systemelemente untersuchen und dabei eine umfassende Sicht auf gesellschaftliche Unsicherheiten und Risiken verlieren. Sie nahmen für die 90er Jahre an, dass sich die Wissenschaft in einer umfassenden "Transition" befindet, einhergehend mit verstärkter Partizipation und zunehmender Toleranz von Diversität hinsichtlich der zum Einsatz kommenden Methoden (Funtowicz \& Ravetz 1993). Das Konzept wurde unter dem Schlagwort Post Normal Science, das auf den von Thomas Kuhn 1962 eingeführten Begriff der Normal Science Bezug nahm, bekannt (Kuhn 1962). Ein Jahr später folgte die Arbeit „The New Production of Knowledge: The Dynamics of Science and Research in Contemporary Societies", mit der Gibbons et al. die Diskussion um neue Formen der Wissensproduktion aufgriffen und vertieften (Gibbons 1994). Sie trugen die Begründung einer radikalen Transformation der Wissensproduktion und des Forschungsprozesses vor und markierten diesen Ansatz mit dem Schlagwort Mode 2. In diesem Konzept findet eine neue Art der Wissensproduktion statt, in der Gesellschaft, Markt und weitere Akteure direkte Bestandteile der Wissensproduktion sind, die in starkem Maße kontextualisiert und durch Transdisziplinarität gekennzeichnet ist.

Drei damals immer offensichtlicher gewordene Trends gaben Anlass, das traditionelle Wissenschaftsverständnis, einhergehend mit der Annahme einer weitgehenden Autonomie der Wissenschaftler und ihrer Institutio- nen, zu hinterfragen. Es wurde erstens deutlich, dass Forschung in zunehmendem Maße über das externe Setzen von Forschungsprioritäten gesteuert wird. Zweitens schritt die Kommerzialisierung der Forschung weiter fort und damit die Verschränkung von Wissenschaft und Wirtschaft. Drittens schließlich rückte die Problematik der Rechenschaftspflichtigkeit und Verantwortlichkeit von Wissenschaft und Forschung gegenüber der Gesellschaft in den Fokus der öffentlichen Debatte. Für das Konzept Mode 2 waren und sind, trotz wissenschaftlicher Kritik an ihm, die folgenden Ansätze konstitutiv:

1. Hervorgehoben wird die Rolle des Anwendungskontextes für Forschung, in dem ein wissenschaftliches Problem entsteht und darauf bezogene Methodologien entwickelt, Ergebnisse disseminiert und Nutzen definiert werden (Nowotny et al. 2003: 186). Darüber hinaus erweiterten sie ihren Ansatz um den Begriff der Agora, die als problemgenerierende und problemlösende Umwelt verstanden wird, in der die Kontextualisierung der Wissensproduktion stattfindet. Die Agora wird verstanden als "domain of primary knowledge production through which people enter the research process, and where Mode 2 knowledge is embodied in people and projects" (Nowotny et al. 2003: 192).

2. Im Konzept Mode 2 spielt die Transdisziplinarität eine hervorgehobene Rolle. Darunter wird die Fähigkeit der Mobilisierung verschiedener theoretischer Perspektiven und praktischer Methodologien zur Problemlösung verstanden. Der kreative Akt liegt hierbei in der Kapazität und Fähigkeit, diese Perspektiven und Methodologien zu mobilisieren und zu managen (Nowotny et al. 2003: 186).

3. Hervorgehoben wird die zunehmende Diversität von Orten der Wissensproduktion und Typen von Wissen, insbesondere auch im Zusammenhang mit neuen Technologien der Kommunikation und Interaktion (Nowotny et al. 2003: 186). 
4. Forschung wird im Paradigma von Mode 2 hochgradig reflexiv. Forschungsprozesse werden dialogisch, es entsteht eine intensive Kommunikation zwischen Forschenden und Forschungsgegenstand. Eine Konsequenz daraus ist die Notwendigkeit, die Rechenschaftspflichtigkeit/Verantwortlichkeit von Wissenschaft und Forschung zu überdenken. Die Folgen des neu produzierten Wissens können nicht mehr nur außerhalb der Forschung thematisiert werden, weil die Problemlösungsumwelten die Themenauswahl, das Forschungsdesign und die Anwendungen bereits beeinflusst haben (Nowotny et al. 2003: 187).

5. Mode 2 ist mit neuen Formen der Qualitätssicherung verbunden. Peers können nicht mehr zuverlässig identifiziert werden, da disziplinenübergreifend gearbeitet wird und am Forschungsprozess unterschiedliche Akteursgruppen beteiligt sind, etwa Wissensproduzenten unterschiedlichster Couleur, Broker, Disseminatoren und Nutzer. Es gibt für die Qualitätsmessung keine eindeutigen Kriterien mehr, und es ist erforderlich zu lernen, mit multiplen Definitionen von Qualität umzugehen (Nowotny et al. 2003).

Bereits diese knappe Skizzierung des Konzepts Mode 2 verweist auf dessen Aktualität für den organisierten Einbezug von Bürgerinnen und Bürgern in Forschungsprozesse. Hierbei spielen alle fünf konstitutiven Merkmale eine Rolle.

An dieses Konzept schließen neuere wissenschaftliche Arbeiten und forschungspolitische Aktivitäten auf internationaler und nationaler Ebene unter dem Schlagwort Open Science an. Es handelt sich um ein neues $P a-$ radigma, wie Forschung betrieben wird, und um eine Strategie, um Wissenschaft mittels Digitalisierungsmaßnahmen effizienter zu gestalten (Vohland \& Göbel 2017; Franzen 2016). Diese beiden Aspekte kommen auch in der entsprechenden Definition der EU-Kommission aus dem Jahr 2016 zum Ausdruck: “Open Science repre- sents a new approach to the scientific process based on cooperative work and new ways of diffusing knowledge by using digital technologies and new collaborative tools. The idea captures a systematic change to the way science and research have been carried out for the last fifty years: shifting from the standard practices of publishing research results in scientific publications towards sharing and using all available knowledge at an early stage in the research process" (Europäische Kommission 2016: 33). Weiter ausdifferenziert wird das Konzept Open Science in der gegenwärtigen deutschen Diskussion von Riehn und Nentwich durch die Herausarbeitung von vier Dimensionen, mit denen Offenheit bzw. Öffnung von Wissenschaft in Zusammenhang gebracht wird (Riehm \& Nentwich 2017). Offenheit von Wissenschaft bezieht sich demnach (1) auf die formale Kommunikation, wobei der offene Zugang zu wissenschaftlichen Daten, Informationen und Publikationen angesprochen ist. Offenheit bezieht sich (2) auf den Teilnehmerkreis der Kommunikation. Eine Öffnung üblicherweise vertraulicher Kommunikationsprozesse, z. B. in wissenschaftlichen Netzwerken, erzeuge mehr Transparenz und wissenschaftliches Arbeiten werde leichter und diskriminierungsfrei nachvollziehbar. Offenheit betrifft (3) die Wissensproduktion selbst. Es gehe um den Abbau hierarchischer und disziplinärer Grenzen der Wissenschaft. Offenheit bezieht sich (4) schließlich auf den Transfer des erzeugten Wissens, wobei es um Fragen der Wirksamkeit der Anwendung der Ergebnisse von Forschung geht (Riehm \& Nentwich 2017).

Reichmann (2017) bringt in die aktuelle Debatte um Open Science zwei Erweiterungen aus der soziologischen Perspektive ein. Zum einen geht es um die Öffnung von Strukturen des Wissenschaftssystems, die über eine Öffnung des Zugangs zu wissenschaftlichem Wissen im Zuge neuer digitaler Publikationsstrukturen weit hinausgeht. Das betrifft beispielsweise die Frage der Öffnung sozialer Strukturen von wissenschaftlichen Communitys oder auch von Kooperationsstrukturen, die seiner Meinung nach mehr oder weniger offen oder geschlossen sein können (Reichmann 2017: 44). Zum anderen möchte er den in der Wissenschaftssoziologie etablierten Kulturbegriff für Open Science nutzbar machen. Aus seiner Sicht eröffnet die Betrachtung von Open Science als Wissenskultur zusätzliche Perspektiven. Dies bedeutet, die Praxis der Wissenschaften in den Blick zu nehmen, weil auch unterschiedliche Wissenskulturen zur Schließung oder Öffnung von Wissenschaft beitragen können (Reichmann 2017: 44). Eine solche Sichtweise hat zur Konsequenz, dass ein universaler Begriff von Open Science nicht sinnvoll erscheint. Es geht vielmehr um differenzierte Betrachtungen von Open Science in Abhängigkeit von speziellen Praktiken, die auf Wissenskulturen basieren. Das heißt, die Öffnung der Wissenschaft schlägt sich in konkreten Wissenschaftspraxen nieder. Insofern ist Open Science lokal verankert und sozial differenziert. Die strukturellen und kulturellen Kontextbedingungen für Open Science unterscheiden sich und müssen berücksichtigt werden. Auch in dieser Perspektive ergeben sich zahlreiche Schnittmengen zum eingangs vorgestellten Konzept Mode 2.

Franzen nennt in diesem Zusammenhang die folgenden "Denkschulen“, die jeweils unterschiedliche Aspekte der Öffnung von Wissenschaft im Fokus haben. Das betrifft die Zugänglichkeit wissenschaftlichen Wissens (Demokratische Schule), die Gestaltung von Wissenschaft (Pragmatische Schule), die Verfügbarkeit von Wissen und Ergebnissen über webbasierte Plattformen (Schule der Infrastrukturen), die Zugänglichkeit für Bürgerinnen und Bürger (Schule der Öffentlichkeit) sowie die Messung wissenschaftlichen Impacts mit alternativen Bewertungssystemen (Schule der Bewertung) (Franzen 2016: 281). Hier wird erstmalig die Perspektive des Bürgers aufgeführt, andere, bereits angelegte Perspektiven werden von Franzen vertieft.

Diese verschiedenen Perspektiven aufgreifend argumentiert Dickel, wenn er im Zusammenhang mit Open Science eine „Erosion der Wissenschaft als professionell geschlossene Sphäre“ infolge der „Umstellung wissenschaftlicher Kommunikation auf 
elektronisch-digitale Verbreitungswege und der damit verknüpften normativ ausgeflaggten Programmatik einer weitgehenden Öffnung der Wissensrezeption (Open Access) und Wissensproduktion (Citizen Science)“ antizipiert (Dickel 2017). Er hält eine allmähliche Entgrenzung des Wissenschaftssystems für möglich, ähnlich wie Schneidewind, der ein Wissenschaftsverständnis vertritt, in dem Wissenschaft nicht nur Transformationsprozesse in der Gesellschaft beobachtet und beschreibt, sondern diese aktiv mitgestaltet (Schneidewind 2015: 88).

Der Ansatz der Transformativen Wissenschaft wurde erstmalig 2014 von Schneidewind und Singer-Brodowski in die Diskussion gebracht. Er baut auf verschiedenen Arbeiten zur Transdisziplinarität, der Aktions- und Interaktionsforschung und der Forschung zu Mode 2 auf (Schneidewind \& Singer-Brodowski 2014). Berücksichtigung finden im Konzept nach Schneidewind auch Ansätze zu Reallaboren oder der zivilgesellschaftlichen Beteiligung. Insofern handelt es sich um einen Ansatz, der in hohem Maße integrativ ist. Er zielt auf die institutionelle Entgrenzung des Wissenschaftssystems und dessen Öffnung "gegenüber anderen Orten der wissenschaftlichen Wissensproduktion“ (Schneidewind 2015: 89). Mit dieser Entgrenzung ist die institutionelle Re-Justierung des bestehenden Wissenschaftssystems verbunden, um „dieses angesichts massiver gesellschaftlicher Umbrüche in neuer Form auf die Gesellschaft zu beziehen“ (Schneidewind 2015: 91).

Um das Konzept der Transformativen Wissenschaft ist in den vergangenen Jahren eine lebhafte Diskussion entstanden, ähnlich wie um das Konzept Mode 2. Als prominentester Vertreter und umfassendster Kritiker kann wohl Peter Strohschneider angesehen werden. Seine Fundmentalkritik richtet sich insbesondere auf vier Merkmale Transformativer Wissenschaft:

- An erster Stelle steht der Vorwurf der Orientierung am Schema "Problem und Lösung“ (Strohschneider 2014: 179).

Er argumentiert mit der Partialität des Themas (Strohschneider 2014: 180) und reduktionistischen Auswirkungen auf das Wissenschaftsverständnis. Die Kritik gipfelt im Vorwurf der Vereinseitigung des Zusammenhangs von Verstehen und Gestalten, was zu einem Privilegieren der praktischen Nützlichkeit gegenüber anderen, eher indirekten Effekten von Wissenschaft führe (Strohschneider 2014: 180).

- An zweiter Stelle geht es um die Kritik des Konzeptes der Transdisziplinarität.

Der Vorwurf besteht hier darin, dass Gesellschaft durch Mitwirkung zum integralen Bestandteil der wissenschaftlichen Wissensproduktion würde. Infolge dessen käme es zu einer Substitution der Strukturierungsleistungen einer disziplinären Wissenschaftsordnung (Strohschneider 2014: 181).

- Drittens geht es in der Kritik um den Status wissenschaftlichen Wissens.

Dieser Status bestimme sich im Ansatz der Transformativen Wissenschaft weniger im Rahmen eines wissenschaftlichen Wahrheitsdiskurses als vielmehr im Nützlichkeitsbereich (Strohschneider 2014: 181). Hervorgehoben wird auch das der Wissenschaft immanente Prinzip des methodischen Zweifels, das die Unterscheidung zwischen wissenschaftlichem und nicht wissenschaftlichem Wissen ermögliche. Strohschneider beschreibt in diesem Zusammenhang unter Bezugnahme auf Hoffmann (Hoffmann 2013) die Gefahr, dass im Falle des Außerkraftsetzens des Prinzips des methodischen Zweifels das irreversible Faktum, das auf dauerhafte Anerkennung pocht, im Modus Transformativer Wissenschaft an Macht gewinnt (Strohschneider 2014: 181).

- Viertens wendet er sich gegen das Argument der Ent-Differenzierung.

Seiner Meinung nach wird im Ansatz der Transformativen Wissenschaft das Prinzip der funktionalen Differenzierung nicht als Möglichkeitsbedingung verstanden, sondern als krisenhaftes Problem moderner Gesellschaften be- schrieben (Strohschneider 2014: 182). Im Vordergrund des Konzepts stehe die Entgrenzung des Wissenschaftssystems statt komplizierter Grenzverhandlungen zwischen Wissenschaftssystem und anderen gesellschaftlichen Teilbereichen (Strohschneider 2014: 182). Schließlich sieht Strohschneider aufgrund der Entdifferenzierung die Gefahr der Einschränkung des Raums des Politischen und dessen Verdrängung durch Wissenschaft, da die Transformative Wissenschaft den Anspruch erhebe, die Transformation der Gesellschaft selbst zu betreiben.

In den Repliken auf diese Kritik, vorgetragen von Schneidewind und Grunwald, wird, neben der Bezugnahme auf die einzelnen Kritikpunkte, an zentraler Stelle darauf verwiesen, dass es der Transformativen Wissenschaft keineswegs darum gehe, das gesamte traditionelle Wissenschaftssystem durch ein neues, an der Nützlichkeit orientiertes und von der Wissenschaftlichkeit abgekehrtes Paradigma zu ersetzen, sondern dass es um notwendige Erweiterungen und eine Neujustierung des Systems gehe, wobei global ohnehin nicht von einer einheitlichen Wissenschaft ausgegangen werden könne (Grunwald 2015; Schneidewind 2015). Grunwald zieht in diesem Zusammenhang die Parallele zum Entstehen der Technikwissenschaften und zu deren erfolgreicher Durchsetzung und Etablierung im Wissenschaftssystem im 19. Jahrhundert, ohne dieses zu ersetzen.

\subsection{Citizen Science als „Spezialfall“ von Open Science}

Wurden im ersten Teil zum konzeptionellen Hintergrund grundsätzliche wissenschaftstheoretische und wissenschaftspolitische Diskussionen der Öffnung des Wissenschaftssystems als ein Diskussionsstrang skizziert, werden im Folgenden Ansätze beschrieben, die stärker auf die steigenden Ansprüche von Akteuren (insbesondere Bürgerinnen und Bürgern) auf Beteiligung in unterschiedlichen Teilbereichen der Gesellschaft generell und an der Wissenschaft im Speziellen fokussieren. Dabei geht es nicht so sehr um die Frage der Ausdifferenzierung unterschiedlicher gesellschaftlicher Teilbereiche. Es geht vielmehr darum, in 
welcher Weise und in welchem Maße diejenigen Menschen partizipieren können, die nicht zu den die Teilbereiche unmittelbar konstituierenden Leistungsträgern gehören. Damit rückt, auch bezogen auf das Teilsystem Wissenschaft, die Akteursperspektive in den Vordergrund (Vohland \& Göbel 2017). Es handelt sich hierbei um emanzipatorische Fragestellungen zur Mobilisierung alternativer Formen von Expertise. Bezieht man Beteiligung auf die Einbeziehung von Nichtwissenschaftlern in Forschungsprozesse, ist man beim Konzept von Citizen Science als einem „Spezialfall“ von Open Science angelangt.

Für das Verständnis von Citizen Science sind Ansätze aus der Rollen- und Professionalisierungstheorie interessant (Mayntz 1988; Stichweh 1988). So beschreibt beispielsweise Gerhards in seinem bekannt gewordenen Artikel „Der Aufstand des Publikums“ aus dem Jahr 2001 die sich verändernde Rolle des Publikums seit den 60er Jahren des 20. Jahrhunderts bis 1989 in verschiedenen gesellschaftlichen Teilsystemen. Er greift hierbei auf den systemtheoretischen Ansatz von Luhmann zurück und verbindet inn mit der o. g. Rollen- und Professionalisierungstheorie sowie der Organisationssoziologie. Der Ausgangspunkt der für Citizen Science relevanten Überlegungen besteht in der Annahme, dass Teilsysteme einer strukturellen Absicherung durch spezifische Rollen bedürfen (Mayntz 1988: 20). An erster Stelle sind hier die sogenannten Leistungsrollen zu nennen. „Mediziner, Pädagogen, Politiker, Juristen, Künstler, Sportler, Wissenschaftler sind Berufsrollenträger innerhalb der jeweiligen Teilsysteme, die die Handlungsrationalität zum Ausdruck bringen und damit strukturell absichern“ (Gerhards 2001: 165). Für viele gesellschaftliche Teilsysteme wie Wirtschaft oder Politik haben sich jedoch nicht nur Leistungsrollen ausgebildet, sondern auch komplementär hierzu Publikumsrollen entwickelt. „Diese Publikumsrollen ermöglichen die Inklusion der Gesamtbevölkerung in die Teilsysteme und definieren die Möglichkeit der Partizipation der Bevölkerung an den verschiedenen Teilsystemen“ (Gerhards 2001: 166). Nach Gerhards hängen die Partizipationschancen von bestimmten Rollenerwartungen ab. Relevant sind allein die Fähigkeiten einer Person, die aus der Sicht eines Teilsystems durch die Leistungsrollen als relevant definiert werden. Das Publikum erhält deshalb Zugang zum Teilsystem erst über den selektiven Zugriff durch die Leistungsträger (Gerhards 2001: 166).

Basierend auf diesem theoretischen Konstrukt weist Gerhards, zumindest für einen bestimmten Zeitraum, nach, dass sich das Verhältnis von Leistungsund Publikumsrollen im Zeitverlauf gewandelt hat. „Die Rechte und Inklusionsansprüche der Laien sind im Verhältnis zu den Autoritätsrollen in fast allen gesellschaftlichen Teilbereichen gestiegen, die Reduktion auf einen recht selektiven Rollenzuschnitt ist aufgeweicht worden, Bürger melden sich als Personen zu Wort, ein Aufstand des Publikums hat stattgefunden" (Gerhards 2001: 167). In seinem Beitrag beschreibt er den Prozess der Umcodierung (Umkehr einer binären Leitdifferenz) des Verhältnisses von Publikumsund Leistungsrollen in verschiedenen Teilbereichen, so auch im Bereich der Politik. Hier ist das Publikum durch die Rolle als Staatsbürger in das politische System eingebunden (1) durch die Möglichkeit der Mitbestimmung über relevante Themen und die Möglichkeit, Träger der Leistungsrollen auszuwählen (Inputseite). Eingebunden ist es aber auch (2) als Abnehmer der getroffenen Entscheidungen (Outputseite). Das Publikum kommuniziert mit den Vollzugsorganen des politischen Systems (Gerhards 2001: 175). Gerhards zeigt dezidiert auf, wie sich seit den 1960er Jahren sowohl auf der Inputseite die geforderten Mitsprachemöglichkeiten des Publikums deutlich erweitert haben als auch auf der Outputseite Inklusionsansprüche der Bürger gestiegen sind. Im Ergebnis, so seine Schlussfolgerung, wurde die Idee eines aktiven Bürgers in die Handlungsrationalität des politischen Systems eingebaut. Das Wissenschaftssystem wurde in diesem Zusammenhang von Gerhards als gesellschaftlicher Teilbereich, der für eine zunehmende Inklusion von Bürgerinnen und Bürgern relevant ist, ausgespart - sicher auch deshalb, weil in differenzierungstheoretischer Sicht das Wissenschaftssystem durch besondere Merkmale gekennzeichnet ist, die den
Zugang erschweren.

Vor einem ähnlichen theoretischen Hintergrund diskutieren Dickel und Franzen 2015 das Problem der Demokratisierung von Wissensproduktion und Wissensrezeption (Dickel \& Franzen 2015) und schließen damit in gewisser Weise die "Lücke“ von Gerhards. Ihre Arbeit wird von der These geleitet, dass sich Konturen neuer wissenschaftlicher Inklusionsprofile herausbilden, die auf ein alternatives Publikum und die Inklusion von Nichtwissenschaftlern in die Wissensproduktion hinweisen. Sie knüpfen an die differenzierungstheoretisch begründete Geschlossenheit des Wissenschaftssystems an, gehen aber davon aus, dass diese sich praktisch aufzulösen scheint.

Auch Dickel und Franzen verweisen zunächst auf Leistungsrollen, die teilsystemspezifisch an Professionsgemeinschaften und die Mitgliedschaft in Organisationen gebunden sind, auf der einen Seite und auf Publikumsrollen auf der anderen Seite. Darüber hinaus referenzieren sie auf die Ausprägung sekundärer Leistungsrollen, wie sie beispielsweise in der Funktion des Kritikers oder des Amateurs zum Ausdruck kommen. Im klassischen differenzierungstheoretischen Verständnis ist Wissenschaft durch die Besonderheit gekennzeichnet, dass es keine konstitutiven Publikumsrollen gibt. Vereinfacht gesprochen ist die Wissenschaft sich ihr eigenes Publikum. Laien oder Bürger sind von der Teilhabe am Teilsystem weitgehend ausgeschlossen. Ihnen wird höchstens eine generelle Publikumsrolle zuerkannt. Die Beteiligung von Laien bleibt auf Formen indirekter Inklusion beschränkt und bezieht sich auf Bildungsangebote seitens der Wissenschaft und den Medienkonsum im Zusammenhang mit Wissenschaft (Dickel \& Franzen 2015: 9).

Dickel und Franzen unternehmen nun den Versuch, diese exklusiven Strukturen zu relativieren. Ihrer Meinung nach kristallisieren sich neue, mediatisierte, funktionale Beziehungen zwischen Wissenschaftlern und Nichtwissenschaftlern heraus (Dickel \& Franzen 2015: 2). Mit Verweis auf Weingart gehen sie davon aus, dass die medienvermittelte Inklusion eines generellen Publikums an Stärke gewinnt (Dickel 
\& Franzen 2015: 9). Neue Inklusionsmodi werden insbesondere im wissenschaftlichen Publikationswesen gesehen, was die Autoren zu der These führt, dass sich eine neue, sekundäre Publikumsrolle entwickelt, die allmählich systeminternes Handeln zu konditionieren beginnt. Diese sekundäre Publikationsrolle entwickelt sich einerseits über die Herausbildung eines konstitutiven wissenschaftlichen Publikums und andererseits über die Wiederbelebung des Amateurforschers. Für beide Tendenzen spielen digitale Technologien als Enabler eine wichtiger Rolle (Dickel \& Franzen 2015: 18). Aber auch auf der Seite der Leistungsrollen (etablierter Wissenschaftlerinnen und Wissenschaftler sowie Wissenschaftseinrichtungen) werden ihrer Meinung nach Differenzierungen sichtbar, entweder als bessere Alternative zu den primären Leistungsrollen oder als Dienstleister für diese primären Leistungsrollen. Für beide, Publikumsrollen und Leistungsrollen, nehmen Dickel und Franzen ein Spannungsverhältnis an, das durch die beiden Pole Gegenstrukturalität und Integration gebildet wird. Auf der Seite der Gegenstrukturalität geht es um Alternativen zu Organisationen und Professionen in einem gesellschaftlichen Teilsystem. Für den Bereich der Wirtschaft werden in Anlehnung an Al-Ani als Beispiel Freie Produzenten im Sinne digital organisierter Produktionsgemeinschaften erwähnt (Dickel \& Franzen 2015: 21; Al-Ani 2013). Im Bereich der Wissenschaft verweisen Dickel und Franzen immer wieder auf die sogenannten Biohacker als Communitys, in denen die dezentralen Infrastrukturen von Bürgerforschern selbst getragen werden (Dickel \& Franzen 2015: 22). Am Pol der Gegenstrukturalität entstehen so alternative Strukturen der Wissensproduktion jenseits etablierter wissenschaftlicher Institutionen, auch wenn die Autoren dies eher als seltene Nischen verstehen und damit relativieren. Am Pol der Integration entwickeln Laien spezifische Publikumsrollen unterschiedlichen Integrationsgrades, vom Datensammler bis zum Mitarbeiter in anwendungsorientierten Forschungsprojekten, und werden so produktiv für wissenschaftliche Organisationen bzw. für primäre Leistungsrollenträger (Dickel
\& Franzen 2015: 27).

Die Autoren lösen sich schließlich vom klassischen differenzierungstheoretischen Systemkonzept, indem sie davon ausgehen, dass die traditionelle Form der funktionalen Differenzierung durch technisch vermittelte Netzwerkstrukturen überlagert wird, die Integrations- und Koordinierungsfunktionen übernehmen. Sie nähern sich insofern den eingangs genannten Open-Science-Konzepten an, ohne jedoch so weit zu gehen, von einer Demokratisierung der Wissenschaft zu sprechen. Sie gehen damit über das ursprüngliche und in der Diskussion auch immer wieder durchscheinende Verständnis von Citizen Science hinaus, in dessen Mittelpunkt Unterstützungsleistungen für die Wissenschaft in der Datenaufnahme und Datenanalyse stehen (Vohland \& Göbel 2017) und Citizen Science lediglich als Form der Wissenschaftskommunikation und -bildung ausgewiesen wird.

Insgesamt hat die Diskussion um Citizen Science ein Stadium erreicht, in dem der Übergang hin zu einem Verständnis, dem zufolge sich Bürgerinnen und Bürger in zunehmendem Maße direkt an Forschungsprozessen beteiligten, vollzogen ist (Häußermann \& Heidingsfelder 2017). Sie fungieren in diesem Verständnis nicht nur als Beobachterinnen und Beobachter oder Sammlerinnen und Sammler von Daten, sondern werfen selbst Forschungsfragen auf und untersuchen diese gemeinsam mit Wissenschaftlerinnen und Wissenschaftlern. Entsprechend dominieren sehr allgemeine, aber auch zum Teil breite Definitionen von Citizen Science. Sanz et al. beispielsweise definieren Citizen Science wie folgt: "Citizen Science refers to the general public engagement in scientific research activities when citizens actively contribute to science either with their intellectual effort or surrounding knowledge or with their tools" (Sanz et al. 2015).

Im deutschen Grünbuch zu Citizen Science wird ein sehr breites Verständnis von Citizen Science vertreten. Als Merkmale von Citizen Science werden hier genannt:

- „Aufnahme zivilgesellschaftlicher Fragestellungen in die Wissenschaft und Förderung der
Handlungsfähigkeit der Teilnehmenden, um als Gemeinschaft zu agieren und auch auf zukünftige Herausforderungen gemeinsam reagieren zu können

- Hinwendung zu problemorientierten Lösungsansätzen auf der Basis von vernetzten wissenschaftlichen und gesellschaftlichen Erkenntnissen auf lokaler, regionaler, nationaler und internationaler Ebene

- Möglichkeit zur Generierung und Verdichtung großskalierter qualitativer und quantitativer Datensätze und Erkenntnisse über große Gebiete oder längere Zeiträume

- Erhebung von Informationen und Zusammenhängen aus den verschiedenen Fachrichtungen

- Vertiefung des Verständnisses für Wissenschaft und Forschung in der Bevölkerung

- Möglichkeit zu zivilgesellschaftlichem Engagement und gesteigerten Mitsprachemöglichkeiten der Bevölkerung bei Anliegen von Wissenschaft und Forschung

- Austausch und Zugang zu Wissen für die wissenschaftliche und gesellschaftliche Gemeinschaft

- Stärkung eines Gemeinschaftsgefühls“ (GEWISS 2016)

Eine besondere Facette in der Diskussion um Bürgerwissenschaft bilden Hochschulen. Schneidewind verweist darauf, dass sich eine starke Bürgerwissenschaft dann besonders gut entwickeln kann, wenn es enge Verknüpfungen mit Hochschulen gibt. Dies hat mehrere Gründe: Hochschulen sind (1) Hotspots für junge, offene und neugierige studierende Bürgerinnen und Bürger mit gesellschaftlich relevanten Fragestellungen, die zum Ausgangspunkt für Forschung gemacht werden können und das Potenzial besitzen, disziplinäre Grenzen zu überschreiten. Hochschulen sind (2) regional verortet und haben eine wichtige Entwicklungsfunktion für 
ihr regionales Umfeld. Diese Funktion ist nicht nur auf den unmittelbaren ökonomischen und technologischen Transfer in die Wirtschaft bezogen. Unter dem Schlagwort "Third Mission" haben Hochschulen auch eine Funktion im Hinblick auf den Transfer von Wissen in weitere regionale Kontexte wie etwa Verwaltung, Bildung und Kultur, Wohnen und Mobilität. In Hochschulen ist es (3) möglich, "den Methodentransfer weit über naturwissenschaftliche Beobachtungsmethoden auszuweiten. Die Vermittlung von Methoden der empirischen Sozialforschung, von ethnographischen Feldzugängen, von ökonomischen Modellen - all das lässt sich in der gemeinsamen Bearbeitung von Citizen Science-Fragestellungen entwickeln. Es eröffnet auch Perspektiven einer erweiterten Form von, offener Hochschule;, in der Bürgerwissenschaftler Angebote zum Methodenerwerb wahrnehmen können“ (Schneidewind 2014).

Schneidewind verweist auch nachdrücklich darauf, dass neben einzelnen Bürgern zivilgesellschaftliche Organisationen wichtige Partner für Bürgerwissenschaft sind und Hochschulen deren Potenzial durch einen institutionalisierten Austausch mit der organisierten Zivilgesellschaft stärker nutzen sollten (Schneidewind 2014).

1.3 Bürgerbeteiligung an politischen Entscheidungsprozessen

Ein weiterer Diskussionsstrang der für die Konzipierung eines bürgerwissenschaftlichen Panels von Interesse ist, ist der der Bürgerbeteiligung, wobei die Beteiligung von Bürgerinnen und Bürgern an politischen Entscheidungsprozessen gemeint ist. Auch wenn es hier um Beteiligung in einem anderen gesellschaftlichen Teilsystem als dem der Wissenschaft geht, lassen sich möglicherweise Erfahrungen aus diesem Teilbereich für den der Wissenschaft und die Konzipierung und den Betrieb eines bürgerwissenschaftlichen Panels nutzen.

Nach Pfennig und Bennighaus erfolgte die Partizipation an politischen Entscheidungsprozessen früher im Wesentlichen über formale (Parteien) und informale Wege (Bürgerinitiativen) (Pfenning \& Benninghaus 2009).
Bei Bürgerbeteiligung im Sinne von partizipativer Demokratie bzw. deliberativer Demokratie hingegen stehen heute weniger direktdemokratische Ansätze oder formalrechtliche Anforderungen aus Verwaltungsverfahren an die Beteiligung von Betroffenen im Vordergrund (Nanz \& Fritsche 2012: 2). Vielmehr geht es „um den Erhalt und die Rückgewinnung politischer Handlungsspielräume sowie um die Schaffung neuer Orte deliberativer Mitbestimmung " (Nanz \& Fritsche 2012: 11). Die Besonderheit neuer Formen der Bürgerbeteiligung besteht darin, dass in dialogischen Verfahren Bürger, zivilgesellschaftliche Akteure und Entscheidungsträger frühzeitig zusammengebracht werden (Nanz \& Fritsche 2012: 12). Zentral ist dabei ein zeitweise projektbezogenes Engagement im losen institutionellen Rahmen auf der Basis freiwilliger Bereitschaft. Es gebe ein Mosaik institutioneller Optionen und individueller Opportunitäten für eine derartige Beteiligung.

Beobachtbar ist weiterhin ein Trend hin zur lokalen und zeitlich begrenzten Interessenwahrnehmung von Bürgerinnen und Bürgern (Pfenning \& Benninghaus 2009: 2). Formen des bürgerschaftlichen Engagements sind zunehmend themenbezogen, zeitlich begrenzt, weniger an politische Parteien gebunden und direkter auf persönliche Interessen zugeschnitten (Nanz \& Fritsche 2012: 11). Hieran anknüpfend lassen sich Bürgerpanels als spezifische Verfahren der Bürgerbeteiligung herausstellen (Nanz \& Fritsche 2012). Anknüpfend an ein Panelverständnis der empirischen Sozialforschung wird ein Bürgerpanel als eine regelmäßig stattfindende Befragung repräsentativ ausgewählter Bürgerinnen und Bürger verstanden. Während Citizens' Panels in Großbritannien weit verbreitet sind, sind sie in Deutschland nach Klages jedoch kaum bekannt (Klages et al. 2008). Bürgerpanels kommen in unterschiedlichen Anwendungsbereichen, etwa der Kommunalpolitik, zum Einsatz. Als Beispiel kann das Modellprojekt Bürgerpanel des Deutschen Forschungsinstituts für öffentliche Verwaltung (FöV) Speyer dienen, das in vier Städten umgesetzt wurde. Zusammenfassend lässt sich ein Bürger- panel verstehen als

- ein Instrument oder eine Form der Bürgerbeteiligung neben Bürgerversammlungen, Foren, oder Nachbarschaftsprojekten,

- ein Format, das auf die Beteiligung an politischen, insbesondere kommunalen Entscheidungsprozessen in unterschiedlichsten gesellschaftlichen Handlungsfeldern (z. B. Umwelt, Kultur, Alter, Schule) zielt,

- ein übergreifender Ansatz, um Begrenzungen anderer Formen zu reduzieren (d. h. geringe Beteiligungsschwellen zu haben, große Teile der Bevölkerung zu erreichen),

- ein Instrument zur Verbesserung der Informationsversorgung der demokratisch gewählten Interessenvertretungen sowie von Verwaltungen und schließlich als

- ein Mittel zum Eröffnen neuer Kommunikationsmöglichkeiten zwischen Politik und Bürger (Stiftung Mitarbeit).

\section{Spezifikationen für ein \\ bürgerwissenschaftliches Panel}

Bürgerwissenschaft hat zahlreiche Gemeinsamkeiten mit Open Science oder Transformativer Wissenschaft (s. Abschnitt 1). Gleichwohl sind auch kritische Einwände und Bedenken gegenüber dem Konzept oder seiner Überhöhung für die Konzipierung bürgerwissenschaftlicher Verfahren zu bedenken. Dies betrifft insbesondere die Problematik der Sicherung wissenschaftlicher Qualität. Für die Konzipierung bürgerwissenschaftlicher Vorhaben ist auch die weiter oben aufgegriffene Idee des Spannungsfeldes zwischen den beiden Polen Gegenstrukturalität und Integration von großer Relevanz. Prinzipiell können bürgerwissenschaftliche Vorhaben entlang der gesamten Achse, die sich zwischen Integration und Gegenstrukturalität aufspannt, angesiedelt werden. Im Zusammenhang mit dem Gedanken der Qualitätssicherung wird sich bürgerwissenschaftliche For- 
schung jedoch wahrscheinlich nicht vorrangig am Pol der Gegenstrukturalität etablieren. Die Zusammenarbeit mit Wissenschaftlerinnen und Wissenschaftlern sowie mit wissenschaftlichen Institutionen dürfte eine Leitplanke für entsprechende Vorhaben sein. Ein Panel wird in diesem Zusammenhang als ein Instrument der Beteiligung von Bürgerinnen und Bürgern an durch Wissenschaftlerinnen und Wissenschaftler geplanten und durchgeführten Forschungsprozessen verstanden.

Anknüpfend an die Vorstellung, dass es Transformativer Wissenschaft um die Lösung gesellschaftlicher Probleme gemeinsam mit Wissensträgern unterschiedlicher „Herkunft“ geht, geraten Forschungsprojekte in den Blick, die einen Anwendungsbezug haben. In vielen Fällen sind solche problemlösungsbezogenen Vorhaben interdisziplinär angelegt und finden ihre transdisziplinäre Erweiterung durch die Einbindung von Akteuren aus dem Anwendungskontext und/oder von Bürgerinnen und Bürgern mit unterschiedlichen Wissenshintergründen, seien sie aus dem Bereich des Alltagswissens und der Alltagserfahrung oder auch aus dem der jeweiligen Profession.

Die Beteiligung von Bürgerinnen und Bürgern ist auch bei Vorhaben ohne direkten Anwendungsbezug sinnvoll und möglich, etwa wenn es um die Unterstützung der Wissenschaft bei der Erhebung von Daten geht, was Finke als Citizen Science light bezeichnet (Finke 2014). Hier standen bisher naturwissenschaftliche Fragestellungen im Mittelpunkt. Aktuelle Beispiele aus der BMBF-Projektlandschaft sind die Unterstützung bei der Beobachtung heimischer Tier- und Pflanzenbestände oder neue Messmöglichkeiten für Umweltparameter. Auch die Sammlung von Daten zum Landleben sowie die Analyse der Plastikbelastungen von Sedimenten sind Beispiele für aktuell geförderte Citizen-ScienceProjekte des Zuschnitts Citizen Science light (Wissenschaft im Dialog o. J.).

Diese Art der Bürgerwissenschaft wird allerdings höchstwahrscheinlich nicht den Schwerpunkt der Beteiligung von Bürgerinnen und Bürgern im konkreten Fall einer Hochschule mit starker ingenieur-wissenschaftlich-technischer, ökonomischer/managementseitiger und verwaltungsorientierter Ausrichtung bilden. Hier rücken eher Themen in den Vordergrund, die auf die Lösung gesellschaftlicher Problemlagen gerichtet sind und eine direkte Einbeziehung der Bürgerinnen und Bürger als sinnvoll erscheinen lassen. Auch hier gibt es Projektbeispiele, die derzeit vom BMBF gefördert werden. In einem dieser Projekte geht es um die Frage, wie Hörhilfen für ein selbstbestimmtes Hören aussehen, sich anhören und anfühlen sollen. In einem anderen werden offene Innovationslabore in den Bereichen Sensorik, virtuelle Realität und Nachhaltigkeitskommunikation etabliert, in denen sich Bürgerinnen und Bürger an der Formulierung relevanter Forschungsfragen beteiligten können. Strategien für eine bessere Nahrungsmittelproduktion in der Stadt und die Erfassung der lokalen Lebensqualität in Quartieren sind Beispiele für Projekte mit starkem Regionalbezug. Auch die Frage, wie soziale Praktiken des Reparierens und Selbermachens erfolgreich angeeignet und in den Alltag der Menschen integriert werden können, ist kürzlich Gegenstand eines Citizen-ScienceProjektes des Zuschnitts Citizen Science proper geworden (Wissenschaft im Dialog o. J.).

Diese letztgenannten Beispiele zeigen den Suchraum auf, der für Projekte eines bürgerwissenschaftlichen Panels besonders geeignet ist, ohne Projekte der Art Citizen Science light von vornherein auszuschließen. Natürlich sollten bürgerwissenschaftliche Panels für unterschiedliche Arten von Befragungen zur Verfügung stehen, ebenso für das Usability Testing von wissenschaftlich-technischen Leistungsangeboten. Sie können sich an Kreativmethoden in Lab-Umgebungen zur Ideengenerierung ebenso beteiligten wie an der Konzipierung und Umsetzung von Prototypingstrategien.

Um die Möglichkeit der Einflussnahme von Citizen Science auf das Agenda Setting für die Forschung zu veranschaulichen, sei an dieser Stelle auf das EU-Projekt "Citizen and Multi-Actor Consultation“ im „Horizon-2020-Projekt“ verwiesen, an dem 38 Partner aus 30 EU-Ländern teilnahmen, die zwischen Oktober 2016 und Februar 2017 Workshops mit 1.500 Bürgern organisierten. In deren Ergebnis konnten 170 Visionen herausgearbeitet werden, die nach anschließender Bearbeitung und Clusterung zu 23 vorgeschlagenen Forschungsthemen und 40 Empfehlungen an die Politik führten (University of Malta 2017).

Wie eingangs erläutert und wie die vorgenannten Beispiele z. T. zeigen, steht Open Science in engem Zusammenhang mit der Digitalisierung als Enabler. Sie ermöglicht neue Formen der Aufnahme und Gewinnung von Daten durch Bürgerinnen und Bürger, beispielsweise über digitale mobile Endgeräte, die Datenanalyse und -auswertung, die Dokumentation der Analyseergebnisse sowie die Kommunikation zwischen den Beteiligten über räumliche Distanzen hinweg. Aus diesem Grund spielt die Entwicklung einer digitalen Plattform für den Aufbau und Betrieb eines bürgerwissenschaftlichen Panels eine wichtige Rolle. Sie soll den Zugang zu den Beteiligten und die Kommunikation im Prozess ermöglichen. Darüber hinaus spielt sie eine wichtige Rolle für die Ergebnisdokumentation und die Sicherung der Transparenz der Prozesse. Auch Akteure, die nicht zum Panel gehören bzw. nicht in ein Forschungsvorhaben involviert sind, sollen die Möglichkeit des Zugangs zu den entsprechenden Informationen erhalten.

Gleichwohl agiert die Hochschule stets in einem regionalen Umfeld und verfügt über vielfältige Potenziale, um sich an der Lösung regionaler Problemlagen zu beteiligen (Schneidewind 2014; Technische Hochschule Wildau 2017). Aus diesen Gründen sollten insbesondere Bürgerinnen und Bürger aus dem regionalen Umfeld der Hochschule in ein bürgerwissenschaftliches Panel einbezogen werden, was neben den digitalen Aspekten der Kommunikation auch analoge Formate vor Ort ermöglicht. Was die Art der Problemlagen betrifft, so kommen als Themen sowohl spezifisch regionale Problemlagen als auch überregionale Problemlagen in Betracht, jeweils mit spezifischen Zugängen, Akteurseinbindungen, Umsetzungsmöglichkeiten und Verbreitungswegen.

Wenn das bürgerwissenschaftliche Panel als Instrument der Öffnung der Hochschule für bürgerwissenschaftliches Engagement konzipiert wird, so 
sollte es eine möglichst stabile personelle Zusammensetzung aufweisen, damit es nicht nur für die Bearbeitung gleicher Themenstellungen, sondern auch für wechselnde Fragestellungen genutzt werden kann. Dies entspricht weitgehend der ursprünglichen Funktion eines Panels als "gleichbleibender Kreis von Auskunftssubjekten (Personen, Betrieben), bei denen über einen längeren Zeitraum hinweg Messungen (Beobachtung, Befragung) zu gleichen Themen in der gleichen Methode und zu den jeweils gleichen Zeitpunkten vorgenommen werden“ (Wübbenhorst o. J.). Panels können jedoch nicht nur für unterschiedliche Fragestellungen genutzt werden, sondern sie sollten auch in einem Maße flexibel sein, dass sie für spezifische Themenstellungen in ihrer $\mathrm{Zu}$ sammensetzung modifiziert werden können.

In der empirischen Sozialforschung spielt bei Panels häufig die Frage der Repräsentativität eine wichtige Rolle, vor allem bei Befragungen. Bei dem hier beabsichtigten bürgerwissenschaftlichen Panel ist dies nicht der Fall, da das Panel keine Stichprobe darstellt, die befragt werden soll und in ihrer Zusammensetzung den Merkmalen einer Grundgesamtheit möglichst ähnlich sein muss. Das bürgerwissenschaftliche Panel soll nicht nur passiver Adressat von wissenschaftlichen Fragen sein, sondern sich aktiv an bestimmten Forschungsthemen beteiligten. Für die Auswahl und Ansprache von Bürgerinnen und Bürgern spielen daher andere Anforderungen als die der Repräsentativität eine Rolle. Zu nennen wären das Interesse an wissenschaftlichen Fragestellungen und Methoden, das Gespür für die Analyse von Problemen und das Finden von Problemlösungsansätzen, die Aufgeschlossenheit gegenüber Neuem in Kombination mit der Fähigkeit zu Kritik und Selbstreflexion, die hier an vorderer Stelle stehen. Solche Eigenschaften sind auch wichtig, um Ansprüchen an Wissenschaftlichkeit in der Zusammenarbeit mit Teams aus Wissenschaftlerinnen und Wissenschaftlern sowie anderen Akteuren, etwa aus Wirtschaft und Verwaltung, genügen zu können. Das schließt auch die Kommunikationsfähigkeit gegenüber Wissenschaftle- rinnen und Wissenschaftlern ein. Aus ganz praktischen Gründen kommen Anforderungen an Mobilität und digitale Erreichbarkeit hinzu.

Bei der Konzipierung und Umsetzung eines bürgerwissenschaftlichen $\mathrm{Pa}$ nels spielen nicht nur Anforderungen an Bürgerinnen und Bürger eine Rolle, sondern auch Anforderungen an diejenigen Wissenschaftlerinnen und Wissenschaftler, die das Panel im Interesse der Hochschule etablieren und betreiben wollen. An erster Stelle sei die Fähigkeit zur verständlichen Darstellung wissenschaftlicher Inhalte genannt, aber auch die Bereitschaft, mit Laien zusammenzuarbeiten. Hinzu kommt die Notwendigkeit der Einbindung der Panelaktivitäten in die Transferstrategie der Hochschule. Sie ergänzen traditionelle Formen des Technologietransfers und eröffnen ihm zugleich neue Zugänge und Formate, indem sie auf die Einbindung und Zusammenbindung unterschiedlicher Akteursgruppen gerichtet sind. Damit geht einher, dass der Panelbetrieb ein internes Scouting für geeignete Themenfelder ebenso erfordert wie ein externes Scouting.

\section{Fazit}

Bürgerwissenschaftliche Panels in der hier skizzierten Form sind aus der Literatur bisher nicht bekannt. Die genannten Merkmale und Funktionen des Panels machen eine aufwendige Panelpflege erforderlich, auch wenn das Panel nicht so umfänglich wie ein typisches Bürgerpanel im Bereich der Politik ist. Ebenso anspruchsvoll sind die Anforderungen an die Konzeption der Projekte, ihre auf den Prinzipien der Wissenschaftlichkeit basierende Umsetzung sowie die Dokumentation der Ergebnisse. Den Ausgangspunkt für den Panelaufbau sollte ein konsensfähiges Basisthema der Hochschule bilden, wobei interessierte Bürgerinnen und Bürger in die konkrete Ausgestaltung einzubeziehen sind.

Im unmittelbaren Sinn von Citizen Science wäre es wünschenswert, die Öffnung einer Hochschule für Bürgerinnen und Bürger in einem Aktionsforschungsansatz zu konzipieren, in dem dieser Prozess selbst zum Gegenstand wissenschaftlicher Betrachtung der beteiligten Akteure gemacht wird. "The research needed for social practice can best be characterized as research for social management or social engineering. It is a type of action-research, a comparative research on conditions and effects of various forms of social action, and research leading to social action. Research that produces nothing but books will not suffice" (Lewin 1946: 202f.). Zu diesem Ansatz gehört ein hohes Maß an Reflexivität und damit auch die Thematisierung der mit einem bürgerwissenschaftlichen Panel verbundenen Nachteile und Risiken. $\mathrm{Zu}$ nennen sind hier beispielsweise die hohe Selektivität der Auswahl, die geringe Größe und die variierende Zusammensetzung des Panels.

In der Literatur zum Themenfeld Citizen Science wird häufig von einem großen Interesse der Bürgerinnen und Bürger an der Teilhabe an Wissenschaft ausgegangen (Wissenschaft im Dialog o. J.). Einer von Wissenschaft im Dialog durchgeführten Befragung unter 1.004 deutschen Bürgerinnen und Bürgern zufolge waren immerhin ein Drittel bereit, an einem CitizenScience-Projekt mitzuarbeiten (Wissenschaft im Dialog 2014: 3). Ob ein solches Interesse tatsächlich nachhaltig und auch in einzelnen Regionen vorhanden ist, bleibt im Prozess zu prüfen und stellt aus praktischer Sicht das wohl größte Risiko für den Aufbau eines bürgerwissenschaftlichen Panels dar.

Um im weiter oben genannten anschaulichen Bild "komplizierter Grenzverhandlungen zwischen Wissenschaft und Gesellschaft statt Entgrenzung " von Strohschneider zu bleiben, geht es beim Aufbau eines bürgerwissenschaftlichen Panels an einer Hochschule um eine systematische und wissenschaftlich begleitete "Grenzöffnung“. Bürgerinnen und Bürger sind bei geöffneten Grenzen nicht mehr nur auf eine generelle Publikumsrolle festgelegt, sondern sie können in eine neue und spezifische Publikumsrolle als konstitutiv Beteiligte hineinwachsen. 


\section{LITERATUR}

Al-Ani A (2013) Freie Produzenten: Die neuen Organisationsformen der Wirtschaft. In: Al-Ani A (ed) Widerstand in Organisationen. Organisationen im Widerstand. Springer Fachmedien Wiesbaden, Wiesbaden, ISBN: 978-3-531-18789-1, pp 115-141. doi: 10.1007/978-3531-18790-7_5

Baldwin C, Hippel Ev (2011) Modeling a Paradigm Shift. From Producer Innovation to User and Open Collaborative Innovation. Organization Science 22(6):1399-1417. doi: $10.1287 /$ orsc. 1100.0618

Chesbrough HW (2003) Open innovation. The new imperative for creating and profiting from technology. Harvard Business School Press, Boston, Mass. ISBN: 9781578518371

Dickel S (2017) Öffnung für alle. TATuP 26(1-2):55-59. doi: 10.14512/tatup.26.1-2.55

Dickel S, Franzen M (2015) Wissenschaft im digitalen Wandel: Demokratisierung von Wissensproduktion und Wissensrezeption? Discussion Paper SP III 2015-601, Berlin

Europäische Kommission (2016) Open innovation, open science, open to the world. A vision for Europe. Publications Office of the European Union, Luxembourg. ISBN: 978-92-79-57346-0

Finke P (2014) Citizen Science. Das unterschätzte Wissen der Laien, Dt. Erstausg. Oekom-Verl., München. ISBN: 386581466

Franzen M (2016) Open Science als wissenschaftspolitische Problemlösungsformel? In: Simon D, Knie A, Hornbostel S, Zimmermann K (eds) Handbuch Wissenschaftspolitik, 2nd edn. Springer Reference Sozialwissenschaften. Springer Fachmedien Wiesbaden, Wiesbaden, ISBN: 978-3-658-05455-7, pp 279-296

Funtowicz SO, Revetz JR (1993) The Emergence of Post-Normal Science. In: Schomberg R von (ed) Science, Politics and Morality. Scientific Uncertainty and Decision Making. Theory and Decision Library. Springer Netherlands, Dordrecht, ISBN: 978-94-015-8143-1, pp $85-123$

Gerhards J (2001) Der Aufstand des Publikums / The Rebellion of the Citizens. Zeitschrift für Soziologie 30(3):463. doi: 10.1515/zfsoz-2001-0301

GEWISS (2016) Citizen Science Strategie 2020 für Deutschland. Grünbuch

Gibbons M (1994) The new production of knowledge. The dynamics of science and research in contemporary societies, First publ. 1994. Reprinted. Sage Publ, London. ISBN: 0803977948

Grunwald A (2015) Transformative Wissenschaft - eine neue Ordnung im Wissenschaftsbetrieb? GAIA - Ecological Perspectives for Science and Society 24(1):17-20. doi: $10.14512 /$ gaia.24.1.5

Grunwald A (2017) Editorial. TATuP 26(1-2):3. doi: 10.14512/tatup.26.1-2.3

Häußermann JJ, Heidingsfelder M (2017) Offen, verantwortlich und verantwortlich offen. TATuP 26(1-2):31. doi: 10.14512/tatup.26.1-2.31

Hippel Ev (2017) Free innovation. ISBN: 978-0-26203521-7

Hoffmann C (2013) Die Arbeit der Wissenschaften, 1. Aufl. Diaphanes, Zürich, Berlin. ISBN: 978-3-03734423-1

Klages H, Daramus C, Masser K (2008) Bürgerbeteiligung durch lokale Bürgerpanels. Theorie und Praxis eines Instruments breitenwirksamer kommunaler Partizipation. Modernisierung des öffentlichen Sektors, Bd. 32. Ed Sigma, Berlin. ISBN: 978-3-8360-7232-8
Kuhn TS (1962) The Structure of scientific revolutions, 2nd ed enlarged. International encyclopedia of unified science Foundations of the unity of science, ; No. 2. Chicago Univ. Press, Chicago, III. ISBN: 0-226-45803-2

Lewin K (1946) Action Research and Minority Problems. Journal of Social Issues 2(4):34-46. doi: 10.1111/j.1540 4560.1946.tb02295.x

Mayntz R (1988) Funktionelle Teilsysteme in der Theorie sozialer Differenzierung. In: Mayntz R, Rosewitz B, Schimank U, Stichweh R (eds) Differenzierung und Verselbständigung. Zur Entwicklung gesellschaftl. Teilsysteme. Campus-Verl., Frankfurt/Main •New York, ISBN: 3593340305, pp 11-44

Nanz P, Fritsche M (2012) Handbuch Bürgerbeteiligung. Verfahren und Akteure, Chancen und Grenzen. Schriftenreihe / Bundeszentrale für Politische Bildung, Bd. 1200. Bundeszentrale für Politische Bildung, Bonn. ISBN: 978-3838902005

Nowotny H, Scott P, Gibbons M (2003) Introduction. 'Mode 2' Revisited: The New Production of Knowledge. Minerva 41(3):179-194. doi: 10.1023/A:1025505528250

Pfenning U, Benninghaus C (2009) Partizipativer Wandel - methodischer Wandel: Neue und klassische Formen der Bürgerbeteiligung im Vergleich. Newsletter. Newsletter Wegweiser Bürgergesellschaft

Reichmann W (2017) Open Science zwischen sozialen Strukturen und Wissenskulturen. TATuP 26(1-2):43-48. doi: 10.14512/tatup.26.1-2.43

Reichwald R, Piller FT (2009) Interaktive Wertschöpfung. Open Innovation, Individualisierung und neue Formen der Arbeitsteilung, 2nd edn. Lehrbuch. Gabler, Wiesbaden. ISBN: 978-3-8349-9440-0

Riehm U, Nentwich M (2017) Open Science aus Perspektive der Technikfolgenabschätzung. TATuP 26(12):11-17. doi: 10.14512/tatup.26.1-2.11

Sanz FS, Holocher-ErtI T, Kieslinger B, Garcia FS, Silva CG (2015) White Paper on Citizen Science for Europe.

Schneidewind U (2014) Bürgeruniversität spiegelt den Dialogwunsch: Konzept der "Bürgerhochschule“. Ein Katalysator für eine starke Bürgerwissenschaft. Wissenschaftsmanagement(3):20-23

Schneidewind U (2015) Transformative Wissenschaft - Motor für gute Wissenschaft und lebendige Demokratie. GAIA - Ecological Perspectives for Science and Society 24(2):88-91. doi: 10.14512/gaia.24.2.5

Schneidewind U, Singer-Brodowski M (2014) Transformative Wissenschaft. Klimawandel im deutschen Wissenschafts- und Hochschulsystem, 2nd edn. Metropolis-Verl., Marburg. ISBN: 9783731610571

Stichweh R (1988) Differenzierung des Wissenschaftssystems. In: Mayntz R, Rosewitz B, Schimank U, Stichweh $R$ (eds) Differenzierung und Verselbständigung. Zur Entwicklung gesellschaftl. Teilsysteme. CampusVerl., Frankfurt/Main•New York, ISBN: 3593340305

Stiftung Mitarbeit Das Bürgerpanel. https://www.buergergesellschaft.de/mitentscheiden/methoden-verfahren/meinungen-einholen-buergerinnen-und-buergeraktivieren/das-buergerpanel. Accessed 09 Jan 2018

Strohschneider P (2014) Zur Politik der Transformativen Wissenschaft. In: Brodocz A, Herrmann D, Schmidt R, Schulz D, Schulze Wessel I (eds) Die Verfassung des Politischen. Festschrift für Hans Vorländer, Aufl. 2014. Springer Fachmedien Wiesbaden, Wiesbaden, ISBN: 9783658047849, pp 175-192

Technische Hochschule Wildau (2017) Innovation Hub 13. Fast Track to Transfer. http://innohub13.de/. Accessed 09 Jan 2018
University of Malta (2017) CIMULACT - HORIZON 2020 Project. Citizen and Multi-Actor Consultation on Horizon 2020. https://www.um.edu.mt/create/eu_projects/ cimulact. Accessed 09 Jan 2018

Vohland K, Göbel C (2017) Open Science und Citizen Science als symbiotische Beziehung? TATuP 26(12):1824. doi: $10.14512 /$ tatup.26.1-2.18

Wissenschaft im Dialog (2014) Wissenschaftsbarometer

Wissenschaft im Dialog (o. J.) Bürger schaffen Wissen. Die Citizen Science Plattform. http://www.buergerschaffenwissen.de/. Accessed 09 Jan 2018

Wübbenhorst K Panel (o. J.). http://wirtschaftslexikon gabler.de/Archiv/3313/panel-v6.html. Accessed 09 Jan 2018

\section{AUTOREN}

Dr. phil. Frank Hartmann

Prof. Dr. rer. pol. Dana Mietzne

Forschungsgruppe Innovations- und Regionalforschung Technische Hochschule Wildau

E-Mail für Korrespondenz:

frank.hartmann@th-wildau.de

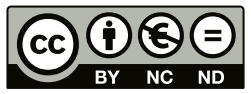

